\title{
Lasers
}

\section{Fusion turned inside out}

from R.G. Evans and M.H. Key

A GROUP of researchers from Osaka University and a compatriot at Bell Laboratories have recently proposed a rather novel approach to controlled fusion (Hasegawa, A. et al. Phys. Rev. Lett. 56, $139 ; 1986)$. They have sought to combine the advantages (or perhaps the pessimists would say, the disadvantages) of both laser-driven inertial confinement and magnetic confinement fusion, and their approach makes use of the highly efficient $\mathrm{CO}_{2}$ laser. This type of laser has been increasingly dismissed as a fusion driver because of its long wavelength, as we recently discussed in these columns (Nature News and Views 313, 94; 1985).

Impressed by the ease with which lasers produce megagauss magnetic fields when they interact with solid targets (for an explanation, see Key, M.H. Nature News and Views 276, 210; 1978), the authors seek to harness these fields in much the same way as a conventional magnetic confinement device such as a Tokamak. The magnetic field $B$ in a Tokamak or mirror

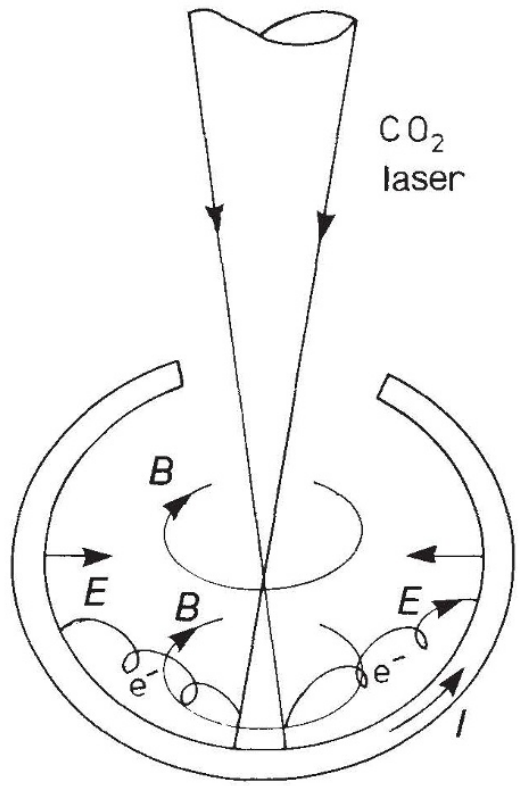

Schematic of Japanese hybrid laser-magnetic confinement target showing $B, E$, electron trajectory $\mathrm{e}^{\prime}$ and current flow $I$.

machine is limited not only by the electrical power needed to drive the magnets but also by the sheer material strength needed to contain the magnetic pressure, which is proportional to $B^{2}$. As the contained plasma pressure cannot exceed the magnetic pressure, this limits the plasma density in a magnetic machine and requires that it contains the plasma for a time of the order of one second for an efficient fusion reaction.

Much effort, particulariy at the Los
Alamos National Laboratory in the United States, has been devoted to devising a fusion scheme that makes use of $\mathrm{CO}_{2}$ lasers, which have the necessary efficiency and energy capability for a fusion driver, but no satisfactory solution has yet been proposed. The present novel target design perhaps offers such a solution.

The figure illustrates the principle in which a $\mathrm{CO}_{2}$ laser is fired into a hollow target. The copious energetic electrons produced by the $\mathrm{CO}_{2}$ laser form current loops (as shown) and produce magnetic fields of several megagauss which spread along the walls as electrons are driven outward by a force caused by perpendicular electric fields $E$ and $B$ (Forslund, D.W. \& Brackbill, J.U. Phys. Rev. Lett. 48, 1614; 1985). Both laser radiation and electron heat conduction inside the cavity ablate material from the inside walls and the relatively cold material flows towards the centre. Here the temperature is higher because of the laser heating and it is suggested that fusion temperatures $(10-100$ million degrees) could be produced. As the density can be quite high, perhaps $10^{21}$ $\mathrm{cm}^{-3}$, because of the enormous magnetic pressure (1 Mbar), the required confinement time is relatively small, $10^{-7} \mathrm{~s}$. Cru- cial to the success of the scheme is the requirement that the magnetic field persists for some time after the laser pulse, and that the central hot plasma is able to refuel itself by ablation of cold material from the inside walls.

Unlike laser fusion there is no need to compress the fuel with the attendant problems of symmetry and fluid instability. There might be magneto hydrodynamic instabilities in the magnetic field that is used to sustain a large temperature difference between the reacting fuel and the walls, but the authors suggest that the system is stable against these effects because of its uniform total pressure.

In support of their rather radical proposals the authors present results obtained with a modest $\mathrm{CO}_{2}$ laser at Osaka University. Using a 1.5-ns laser pulse focused inside hollow plastic spheres up to $3 \mathrm{~mm}$ in diameter they show that the hot plasma lifetime exceeded the duration of the laser pulse by up to 10 times.

It is too early to assess the prospects of this approach to fusion, as many details of the scheme have not been analysed and experiments are at an early stage. But the work is indicative of substantial Japanese involvement in all branches of fusion research and shows their increasing commitment to this research at a time when the United States and Europe are reducing their fusion programmes.

R.G. Evans and M.H. Key are at the Rutherford Appleton Laboratory, Didcot OX11 0QX,UK.

\section{Immunology}

\section{Antigen binding and T cells}

\section{from N.A. Mitchison}

LYMPHOCYTE recognition of antigens is a crucial feature of the immune response and occurs by means of receptor molecules. Although these receptors have fundamentally the same structure on the two major types of lymphocytes they work in very different ways. B cells, which use immunoglobulin molecules as their receptors, can bind antigen without the intervention of other molecules. This is not the case for $T$ cells which normally bind antigen only when it is associated with a glycoprotein encoded by the major histocompatibility complex (MHC) on the surface of another cell. The evolutionary reasons for this 'dual recognition' are by now well understood. It provides a mechanism for ensuring that $\mathrm{T}$ cells come into action only when they touch another cell, which is important because they regulate or kill other cells through cell-to-cell contacts or at short range, unlike B cells whose secretions act far off. Two papers in this issue, Watts, T.H. et al. (Nature 320, $179 ; 1986$ ) and Ashwell, J.O. \& Schwartz,
R.H. (Nature, 320, 176; 1986) take us a step forward in understanding how $\mathrm{T}$ cells recognize antigen.

Any mechanism of this sort needs to be adjusted so that free antigen cannot block the T-cell receptor and MHC molecules do not activate on their own. Part of the adjustment seems to be pre-programmed in the receptor structure, but also selection of suitable receptors occurs in the thymus - hence 'restriction', the ability of $T$ cells to recognize antigen only in the context of the MHC molecules to which they were exposed in the thymus.

Clearly an arrangement of two entirely separate receptors would have difficulty in meeting these requirements and would in any case be incompatible with current knowledge of receptor molecular genetics, so other possibilities have been canvassed. The simplest is a termolecular complex, in which receptor, MHC molecule and antigen all interact with one another. It would still seem difficult to adjust the affinities so as to prevent 